\title{
Hospitalisation for more than 3 days after thrombolysis for uncomplicated myocardial infarction was not cost effective
} Newby LK, Eisenstein EL, Califf RM, et al. Cost effectiveness of early discharge after uncomplicated acute myocardial
infarction. N Engl J Med 2000 Mar 16;342:749-55.

QUESTION: For patients who have had a low risk myocardial infarction (MI), is early discharge on day 3 instead of day 4 after thrombolysis safe and cost effective?

Design

Decision analytic model using data from a randomised controlled trial (Global Utilization of Streptokinase and Tissue Plasminogen Activator for Occluded Coronary Arteries [GUSTO-1]).

\section{Setting}

$\{1081$ hospitals in 15 countries $\}$ *

\section{Patients}

38911 of 41021 enrolled patients who presented with a confirmed MI 20 minutes to 6 hours after symptom onset and who had an uncomplicated course (absence of death, reinfarction, congestive heart failure, recurrent ischaemia, stroke, shock, emergency angiography or angioplasty, bypass surgery, intra-aortic balloon pumping, cardioversion, or defibrillation). Exclusion criteria were angiographic 3 vessel or left main coronary artery disease or elective bypass surgery $>3$ days after thrombolysis.

\section{Intervention}

Discharge at day 3 compared with day 4 after thrombolysis.

\section{Main cost and outcome measures}

Cardiac events on day 4 (reinfarction, stroke, recurrent ischaemia, heart failure, and other major complications requiring readmission) and rate of preventable and non-preventable death. Costs were for hospital resources (including monitoring) and physician fees in 1997 US dollars with a 3\% annual discount rate. Sensitivity analyses were done.

Sources of funding: Bayer; CIBA-Corning; Genentech;ICI Pharmaceuticals; Sanofi

Pharmaceuticals

For correspondence: $\operatorname{Dr} L$ K Newby, Duke Clinical Research Institute, $P O B$ Box 17969, Durham NC 27715-7969, USA Fax +1919668 7059

\section{Main results}

During the 4th day of hospitalisation, 16 patients $(0.4 \%)$ had ventricular arrhythmias, 3 of whom died. These 16 patients were older and more likely to have diabetes and a history of angina or MI. By keeping patients an extra day, 0.006 y $(53 \mathrm{~h})$ of life was saved at a cost of $\$ 624 /$ patient or $\$ 105629$ /life year saved $(95 \%$ CI $\$ 65$ 777 to $\$ 183525)$. Sensitivity analyses showed that a 4 th day in hospital was attractive only if the costs were reduced by $50 \%$ or a high risk subgroup of patients could be identified.

\section{Conclusion}

Admission to hospital of patients with uncomplicated myocardial infarction for $>3$ days after thrombolysis was economically unattractive.

*The GUSTO Investigators. N Engl J Med 1993;329: 673-82.

\section{COMMENTARY}

As pressure to reduce medical costs increases, patients are discharged "quicker and sicker" after surgery and acute medical illnesses. Although longer hospital stays add to the margin of safety, are they worth the cost? Newby et al used a decision model to examine the cost effectiveness of staying in the hospital more than 3 days after an uncomplicated acute MI treated with thrombolysis. In their model, the only benefit of staying longer was preventing sudden death caused by ventricular arrhythmia-a rare event that occurs in approximately 8 of 10000 patients. This benefit was weighed against the cost of staying 1 extra day in the hospital (\$624). Under these assumptions, the longer hospital stay was not cost effective by accepted standards.

The results of this cost effectiveness model are provocative and interesting, but they need to be confirmed in other studies before they can be accepted. Several additional factors must also be considered. Firstly, a longer hospital stay might have additional benefits not considered in the model, such as improved patient education and better initiation of secondary prevention. Fumbling the handoff between inpatient and outpatient care through hasty discharge could easily lead to preventable problems and reduce the net benefit. Secondly, the projected cost savings may be too optimistic because a modest increase in costly readmissions could wipe out the $\$ 624$ per patient saved by early discharge. For example, a $1 \%$ increase in the incidence of complications that cost $\$ 30000$ each to treat would make the longer stays cost effective

Finally, Newby et al did not actually discharge patients after 3 days-they only projected what might happen if this policy were adopted. Very few patients are currently sent home that quickly, and it will be critical to show the actual outcomes of this approach in prospective studies that are large enough to assure that low complication rates are maintained. Until such evidence is available, we should regard the study by Newby et al as hypothesis generating and in need of confirmation.

Mark A. Hlatky, MD Stanford University School of Medicine Stanford, California, USA 